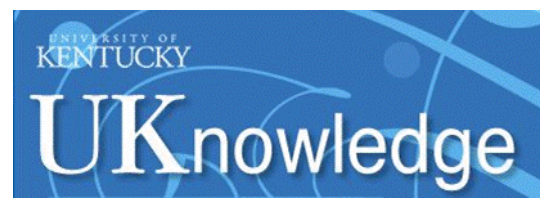

University of Kentucky

UKnowledge

$1-4-2021$

\title{
Numerical Reconstruction of Spalled Particle Trajectories in an Arc-Jet Environment
}

\author{
Raghava S. C. Davuluri \\ University of Kentucky, raghava.sai.chaitanya@gmail.com
}

Sean C. C. Bailey

University of Kentucky, sean.bailey@uky.edu

Kaveh A. Tagavi

University of Kentucky, kaveh.tagavi@uky.edu

Alexandre Martin

University of Kentucky, alexandre.martin@uky.edu

Follow this and additional works at: https://uknowledge.uky.edu/me_facpub

Part of the Aerodynamics and Fluid Mechanics Commons, Fluid Dynamics Commons, Heat Transfer, Combustion Commons, Materials Chemistry Commons, Numerical Analysis and Computation Commons, and the Structures and Materials Commons

Right click to open a feedback form in a new tab to let us know how this document benefits you.

\section{Repository Citation}

Davuluri, Raghava S. C.; Bailey, Sean C. C.; Tagavi, Kaveh A.; and Martin, Alexandre, "Numerical Reconstruction of Spalled Particle Trajectories in an Arc-Jet Environment" (2021). Mechanical Engineering Faculty Publications. 78.

https://uknowledge.uky.edu/me_facpub/78

This Conference Proceeding is brought to you for free and open access by the Mechanical Engineering at UKnowledge. It has been accepted for inclusion in Mechanical Engineering Faculty Publications by an authorized administrator of UKnowledge. For more information, please contact UKnowledge@lsv.uky.edu. 
Numerical Reconstruction of Spalled Particle Trajectories in an Arc-Jet Environment

Digital Object Identifier (DOI)

https://doi.org/10.2514/6.2021-1172

Notes/Citation Information

Published in AIAA Scitech 2021 Forum.

Copyright ( 2021 by Raghava S. C. Davuluri, Sean C. C. Bailey, Kaveh A. Tagavi, Alexandre Martin.

The copyright holders have granted the permission for posting the article here. 


\title{
Numerical reconstruction of spalled particle trajectories in an arc-jet environment
}

\author{
Raghava S.C. Davuluri* Sean C. C. Baileył Kaveh A. Tagavi $\ddagger$ and Alexandre Martin§, \\ University of Kentucky, Lexington, KY, 40506
}

\begin{abstract}
To evaluate the effects of spallation on ablative material, it is necessary to evaluate the mass loss. To do so, a Lagrangian particle trajectory code is used to reconstruct trajectories that match the experimental data for all kinematic parameters. The results from spallation experiments conducted at the NASA HYMETS facility over a wedge sample were used. A data-driven adaptive methodology was used to adapts the ejection parameters until the numerical trajectory matches the experimental data. The preliminary reconstruction results show that the size of the particles seemed to be correlated with the location of the ejection event. The size of the particles ejected from the bottom edge of the wedge varies over three orders of magnitude, whereas the size of the ones ejected from the top (inclined) surface were more uniform (around 10 microns). On the bottom edge, the particles ejected near the leading edge were bulkier (10-1000 microns), where those that ejected further along, had a smaller size (0.1-1 microns).
\end{abstract}

\section{Introduction}

A BLATIVE materials counter the high heat rates during atmospheric re-entry by undergoing mass-removal mechanisms and are used as Thermal Protection System (TPS) for space vehicles. Spallation is one such mechanism, where the mass is removed through solid particles ejections from the material into the flow. The particles are most likely produced by disconnected fibers or chunks of material that are ejected due to thermal, mechanical shear, and internal pressure stresses. They could also be formed by soot, a by-product of the pyrolysis process.

The presence of spalled particles in the flow field affects the surface heating rates and is not a desirable phenomenon. Before the material ablates, the particles eject, which results in escalated surface heat rates and accelerated material recessions as it is equivalent to losing TPS material without the accompanying absorption of the heat. The particles traveling through high-temperature regions tend to react chemically and physically with the species in the flow. It causes a change in the composition of the flow field, thereby modifying the radiative heat flux. The heated and often glowing particles radiate energy back to the material, thus increasing the heat flux. However, the effect of spallation phenomena alone when compared with other ablation processes has not been quantified well. The main reason for that is the difficulty in determining the size of the particles and in understanding the events that lead to particle ejections.

In the past, few experiments were performed to estimate the spallation mass loss from an ablative material. A laser irradiation experiment was conducted by Lundell ${ }^{1}$ to investigate the spallation mass loss on carbon phenolic composites used for Galileo probe fore-body heat shield. A special particle canister ${ }^{2}$ was coupled to a gas-dynamic laser ${ }^{3}$ in which carbon phenolic specimens were mounted. The canister was used to capture the spalled particles during the experiment and were later weighed to evaluate the spallation mass loss. It was found out that the thermochemical mass loss of $7.4 \%$ for the nominal Jovian atmosphere and $10 \%$ for the cold-dense Jovian atmosphere was contributed by spallation. Tests in a hydrogen-helium arc-jet wind tunnel were conducted by Park et. al. ${ }^{4}$ on a carbon phenolic blunt body at Galileo probe heating conditions. There was a disagreement between experimental values and theoretical values given by Radiating Shock

\footnotetext{
*Graduate Student, Mechanical Engineering, AIAA Student Member

${ }^{\dagger}$ Associate Professor, Mechanical Engineering

¥Professor, Mechanical Engineering

§Professor, Mechanical Engineering, Associate Fellow AIAA. alexandre.martin@uky.edu
} 
Layer Environment (RASLE) ${ }^{5}$ and Charring Materials Ablation (CMA $)^{6}$ codes, which was believed to be due to spallation. The spallation rate of about $15 \%$ and $30 \%$ of the total ablation rate was observed for wall heat fluxes of about $20 \mathrm{~kW} / \mathrm{cm}^{2}$ and $30 \mathrm{~kW} / \mathrm{cm}^{2}$. Park ${ }^{7,8}$ also conducted ballistic-range tests on flat disks made of carbon phenolic and carbon-carbon in an argon environment. The experimental results revealed that spallation contributed to the difference between the test and theoretical values, which was $60 \%$ for carbon phenolic and $30 \%$ for carbon-carbon. Also, it was noted that the spallation in carbon phenolic was twice more than for carbon-carbon, and was nearly thrice more than the spallation found by laser tests. The size of the ejected particles was tiny in the carbon-carbon case and ranged from very small to very large for carbon phenolic. The results indicated that the luminosity of particles was relatively constant for the carbon-carbon model and varying for the carbon phenolic model. The tests concluded that spallation due to large particles was only one-tenth of the total spallation as the indentations due to large particles were only $5 \%$ of the total volume of carbon phenolic. Later, another set of ballistic range tests were conducted in an argon environment by Park and Balakrishnan ${ }^{9}$ on carbon phenolic materials used for the Galileo Probe, and it was found that the spallation rate was on the order of $10 \%$ of the total ablation rate.

Recently, a series of spallation experiments and analysis ${ }^{10-13}$ was conducted in an arc-jet environment on a wedge-shaped sample made up of carbon-carbon and carbon-phenolic materials. The trajectories of individual particles were reconstructed through images and using Particle Tracking Velocimetry (PTV) technique. The PTV technique results show that a significant part of spallation happens from the bottom part of the leading edge and the rest from the upper surface. The images' domain was later divided into smaller $1 \mathrm{~mm}^{2}$ regions, and the kinematic parameters were averaged to study the behavior of particles statistically. It was observed that the particles eject with a low velocity and accelerate rapidly to the flow velocity. However, the analysis could not estimate mass loss as the size of the particles was impossible to be measured through the available resolution of the images. Also, the experimental trajectory's initial point was close to the surface and not on the surface. Hence, the experiments could not determine the position and velocity with which the chunks get ejected accurately.

In order to determine the size and other ejection parameters of the particles, a Lagrangian particle trajectory code ${ }^{14}$ is used here to reconstruct the trajectories consistent with the experimental points. A Computational Fluid Dynamics (CFD) code, KATS (Kentucky Aerodynamic Thermal-response Solver), is used to simulate the flow field around the wedge sample. The reconstruction follows a data-driven adaptive methodology and is coupled with the particle trajectory code. The adaptive method tends to minimize the closeness between the numerical and experimental trajectories. The size and other ejection parameters determined through the reconstruction would provide a reasonable estimate for the mass loss due to spallation alone and phenomena behind the particle ejections. The numerically reconstructed trajectories can be later used to study the particle's effect on the flow field. ${ }^{15}$ Preliminary results are provided in this paper, where 600 experimental trajectories were reconstructed, and a statistical analysis of particle's size is studied.

\section{Experimental reconstruction}

Spallation experiments were conducted at the NASA Langley Hypersonic Materials Environmental Test System (HYMETS) by Bailey et al. ${ }^{10}$ The experiment constituted of inserting wedge-shaped samples, made from FiberForm ${ }^{\circledR}$ and PICA, into a Mach 5 flow of air and for different wall heat fluxes for about 30 seconds. High-speed imagery of the sample, as well as the ejected particles, was taken with cameras. One camera was positioned to capture the side-view and another one to capture the bottom-view of the sample. The imaging was performed at a rate of 44,025 frames per second and for a total time of 3.134 seconds by both the cameras. The images were then pre-processed by applying a high-pass filter and subtracting the background illumination mask. Before tracking the path of individual particles, the coordinate system of each image was transformed from camera to laboratory.

The tracking of the particles was performed by using particle tracking velocimetry technique. An opensource software OpenPTV ${ }^{16}$ was used for reconstructing the trajectories. An approximate of 90 particles per frame were identified, and a total of 85000 individual particle trajectories were constructed. Fig. 1 shows a sample of trajectories that have been reconstructed from the images.

The reconstructed trajectories consisted of the kinematic information of spalled particles. However, the initial positions of the particles tracked by the experiments were near the sample. Also, it was difficult to measure the size of the particles due to the low resolution of the images and light emitted by the hot particles that produced bleeding images. Hence, to determine the size and other kinematic information of particles 


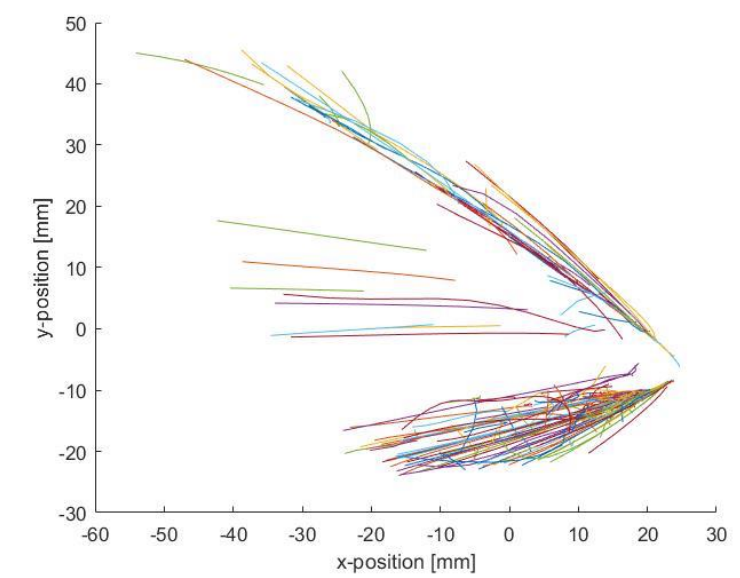

Figure 1. Trajectories reconstructed using PTV technique

ejected from the sample, a numerical reconstruction method is employed that computes trajectories that match the experimental ones in all path-determining parameters of particles. The determination of particle sizes and initial ejection parameters would help estimate the mass loss due to spallation and understanding of the phenomenon behind the ejections.

\section{Numerical approach}

\section{III.A. Lagrangian particle trajectory code}

The model simulates the dynamics of a particle by employing a Lagrangian formulation. ${ }^{17}$ The model also considers the chemical interaction of the particle with the flow field. The governing equation of the model is of the form:

$$
\frac{\partial \mathbf{U}}{\partial t}=\mathbf{W}
$$

where $\mathbf{U}$ is the state vector and $\mathbf{W}$ is the source term vector. The elements of the vector are represented as:

$$
\mathbf{U}=\left(\begin{array}{c}
m_{p} \\
m_{p} u_{p} \\
m_{p} v_{p} \\
m_{p} w_{p} \\
m_{p} E_{p}
\end{array}\right), \quad \mathbf{W}=\left(\begin{array}{c}
\dot{m}_{\text {chemistry }} \\
F_{D_{x}} \\
F_{D_{y}} \\
F_{D_{z}} \\
\dot{q}_{\mathrm{conv}}+p_{\mathrm{drag}}-\dot{q}_{\mathrm{rad}}+\dot{q}_{\mathrm{rxn}}
\end{array}\right)
$$

where $m_{p}$ is the mass of the particle, $\left(u_{p}, v_{p}, w_{p}\right)$ are velocity components of the particle, $E_{p}$ is the total energy of the particle, $\dot{m}_{\mathrm{C}}$ is the chemical reaction source term, $\left(F_{D_{x}}, F_{D_{y}}, F_{D_{z}}\right)$ are components of drag force acting on the particle, and $\dot{q}_{\text {conv }}, \dot{q}_{\text {rad }}, \dot{q}_{\text {rxn }}, p_{\text {drag }}$ are convective, radiative, reaction heat rates, and power required to overcome force acting on the particle. The discretization of the system of equations in Eq. 2 is performed using a backward Euler method. The mass conservation equation is uncoupled from momentum and energy conservation equations, and the Block Gauss-Seidel method is used to solve the two sets of equations. The set comprising of momentum and energy equations is solved using Newtons method. The model is verified using the method of manufactured solution ${ }^{18}$ to confirm the order of accuracy of the discretization and correctness of the numerical code. The flow field data used in the model is extracted from the converged solutions computed by aerothermodynamic computational fluid dynamics (CFD) code KATS (Kentucky Aerothermodynamics and Thermal-response Solver). The model uses a blended drag coefficient model, and is validated by comparing with ten different sets of experimental data. ${ }^{19}$ The model is extensively used to study the spallation phenomenon. A numerical study was conducted in the past to understand how the flow field effects the dynamics of the spalled particles by one-way coupling and the effect spalled particles 
have on the flow field by two-way coupling the numerical code and KATS. More information on these studies are given in Ref. ${ }^{14,15,17,20}$

\section{III.B. KATS - CFD}

The thermo-chemical non-equilibrium flow field in the continuum regime is computed using KATS-CFD, a laminar Navier-Stokes solver. ${ }^{21}$ The governing equation of the model is of form:

$$
\frac{\partial \mathbf{Q}}{\partial t}+\nabla \cdot\left(\mathcal{F}-\mathcal{F}_{\boldsymbol{d}}\right)=\mathbf{S},
$$

where $\mathbf{Q}$ is a vector of conservative variables, $\mathcal{F}$ and $\mathcal{F}_{\boldsymbol{d}}$ are convective and diffusive flux matrices, and $\mathbf{S}$ is the source term vector.

The vectors of conservative variables and source terms are of form:

$$
\mathbf{Q}=\left(\begin{array}{c}
\rho_{1} \\
\vdots \\
\rho_{n g s} \\
\rho_{g} u \\
\rho_{g} v \\
\rho_{g} w \\
\rho E \\
\rho E_{v e}
\end{array}\right), \quad \mathbf{S}=\left(\begin{array}{c}
\dot{\omega}_{1} \\
\vdots \\
\dot{\omega}_{n g s} \\
0 \\
0 \\
0 \\
0 \\
\dot{\omega}_{v e}
\end{array}\right)
$$

where $\rho_{i}$ is the density of species $i,(u, v, w)$ are the components of bulk velocity, $E$ and $E_{v e}$ are the total energy and vibrational-electron-electronic energy per unit mass characterized by temperature $T$ and $T_{v e}$, respectively. $\dot{\omega}_{i}$ is mass production rate of species $i, \dot{\omega}_{v e}$ is the vibrational energy transfer rate between two different energy modes, subscripts from 1 to $n g s$ represent the number of species, and subscript $g$ represents the gas mixture. The convective and diffusive flux matrices in Eq. 3 are given as:

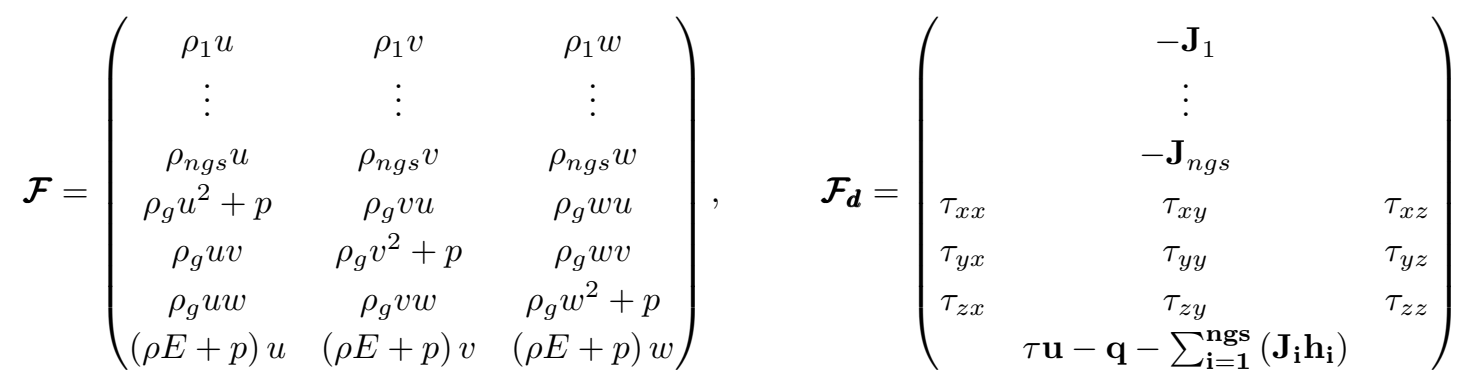

where $p$ is the total pressure, $\tau$ is viscous tensor, $\mathbf{J}_{\mathbf{i}}$ is the diffusive flux of species $i$, and $\mathbf{q}$ is the directional heat flux vector. Eq. 3 is discretized first-order in time and second-order in space. The solver uses PETSc library ${ }^{22-24}$ to solve the linear system of equations, ParMETIS ${ }^{25}$ for domain decomposition, and MPI ${ }^{26}$ for message passing.

\section{III.C. Numerical Reconstruction}

\section{III.C.1. Data Adaptation}

The numerical reconstruction undergoes a data-driven adaptation technique, ${ }^{27}$ the details of which are discussed below. It is an iterative process where adaptation is performed for four parameters that are defined as

$$
\theta=\left[\begin{array}{llll}
\mathrm{r}_{p} & \mathrm{u}_{p} & \mathrm{v}_{p} & \mathrm{w}_{p}
\end{array}\right]^{T}
$$


The elements of the $\theta$ represent radius and velocity components of the particle. The adaptation is performed by minimizing the difference between the simulated and experimental values and the cost function used to define the difference is formulated as

$$
\mathrm{J}(\theta)=\sum_{i=1}^{N}\left[\phi_{n}-\phi_{e, i}\right]^{T} \mathcal{W}_{\mathbf{i}}\left[\phi_{\mathbf{n}}-\phi_{\mathbf{e}, \mathbf{i}}\right]
$$

where $\phi_{n}$ and $\phi_{e, i}$ denote the simulated and experimental position components of the particle and the summation is performed for total number of points $(N)$ of individual experimental trajectory. The experimental data usually has less number of points when compared to the simulated data. Therefore, the difference between $\phi_{n}$ and $\phi_{e, i}$ represent the minimum distance between the simulated curve and $i$ th experimental point. The matrix $\mathcal{W}_{\mathbf{i}}$ is a positive-definite symmetric weighting matrix that is used to express the relative importance of each experimental value and also the relative importance of each adaptive parameter in that experimental value. The weighting matrix is defined as

$$
\mathcal{W}_{\mathbf{i}}=\mathrm{w}_{\mathbf{i}} \mathcal{I}
$$

where $\mathrm{w}_{i}$ is the weight at the $i$ th point of the trajectory and $\mathcal{I}$ is the identity matrix. The weight considered for this case is

$$
\mathrm{w}_{i}=\frac{1}{\left\|\phi_{\mathbf{e}, \mathbf{i}}\right\|^{2}}
$$

where $\|\cdot\|$ is the Euclidean norm. The gradient for the nonlinear cost function $\mathrm{J}$ in Eq. 7 is derived numerically. The approximate gradient is defined as

$$
\Gamma(\theta)=\left[\begin{array}{c}
\frac{\mathrm{J}\left(\theta+\epsilon_{\mathbf{1}}\right)-\mathrm{J}(\theta)}{\epsilon_{1}} \\
\vdots \\
\frac{\mathrm{J}\left(\theta+\epsilon_{\mathbf{4}}\right)-\mathrm{J}(\theta)}{\epsilon_{4}}
\end{array}\right]
$$

where $\epsilon_{i}$ denotes the perturbation of $i$ th element of $\theta$. The perturbations should be very small in order to make the approximate gradient equivalent to $\partial(\mathrm{J}(\theta)) / \partial \theta$. The gradient determines the direction in which the $\theta$ should be adapted in order to reduce the difference between simulated and experimental points. At each iteration step $\mathrm{n} \in \mathbb{N}=\{0,1,2, \ldots\}$, the cost function and its respective gradient is calculated, and the updated $\theta$ is determined as

$$
\theta_{\mathbf{n}+\mathbf{1}}=\theta_{\mathbf{n}}-\xi_{\mathbf{n}} \Gamma\left(\theta_{\mathbf{n}}\right)
$$

where $\xi_{n}$ is defined as adaptive step size. An optimal form of step size is used in this work which is expressed as

$$
\xi_{\mathrm{opt}, n}=\frac{2\left[\mathrm{~J}\left(\theta_{\mathbf{n}}\right)-\mathrm{J}\left(\theta_{*}\right)\right]}{\left\|\Gamma\left(\theta_{\mathbf{n}}\right)\right\|^{2}}
$$

where $\theta_{*}$ is a local minimizer of $\mathrm{J}$ such that

$$
\left\|\theta_{\mathbf{n}+\mathbf{1}}-\theta_{*}\right\|^{2}-\left\|\theta_{\mathbf{n}}-\theta_{*}\right\|^{2}<0
$$

The iterative procedure is continued till the cost functions as defined in Eq. 7 is below the prescribed tolerance value.

\section{III.C.2. Time of Flight}

The adaptation of the numerical trajectory to match the experimental one is performed in two steps. In the first step, the first position $\left(\phi_{n}\right)$ from the simulated trajectory is picked based on its proximity with the first experimental data point. The rest of the simulated trajectory points are selected based on their flight time matching with the experiment. In this way, the numerical trajectory matches the positions as well as the time of flight of experimental points. 


\section{III.C.3. Density of particles}

The other important parameter to be considered for reconstruction is the density of the particles. The spalled particle may be a single fiber or a chunk of fibers. The Lagrangian particle trajectory code assumes the particles to be spherical. A numerical $\operatorname{code}^{28}$ is developed that assumes a spherical volume around the particle. The code reads the micro-CT scan image of fibrous material as an input. The algorithm searches for the fiber center and starts increasing the spherical volume around it by one grid point at each step. The control volume's porosity is calculated at each step, and the control volume is increased until it reaches a critical value. The critical value is defined as the volume at which the porosity is equivalent to the material's porosity. It should also be noted that when the control volume's size is less than or equal to the volume corresponding to the fiber's diameter, the porosity is zero. The code is run for different fiber centers, and an average of the critical value is calculated.

A linear relation is established between the control volume's density and size based on the critical value. If the porosity is zero, then the density of the spherical volume is that of fiber, and if higher than critical value, then it is the density of the material. In this way, the non-sphericity of particles is taken into consideration while simulating the trajectories. The density of single fibers and FiberForm ${ }^{\circledR}$ (whose porosity is 0.87) is taken from the work performed by Panerai et al. ${ }^{29}$ Figure 2 shows the relationship that the particle code takes to calculate the density based on the size.

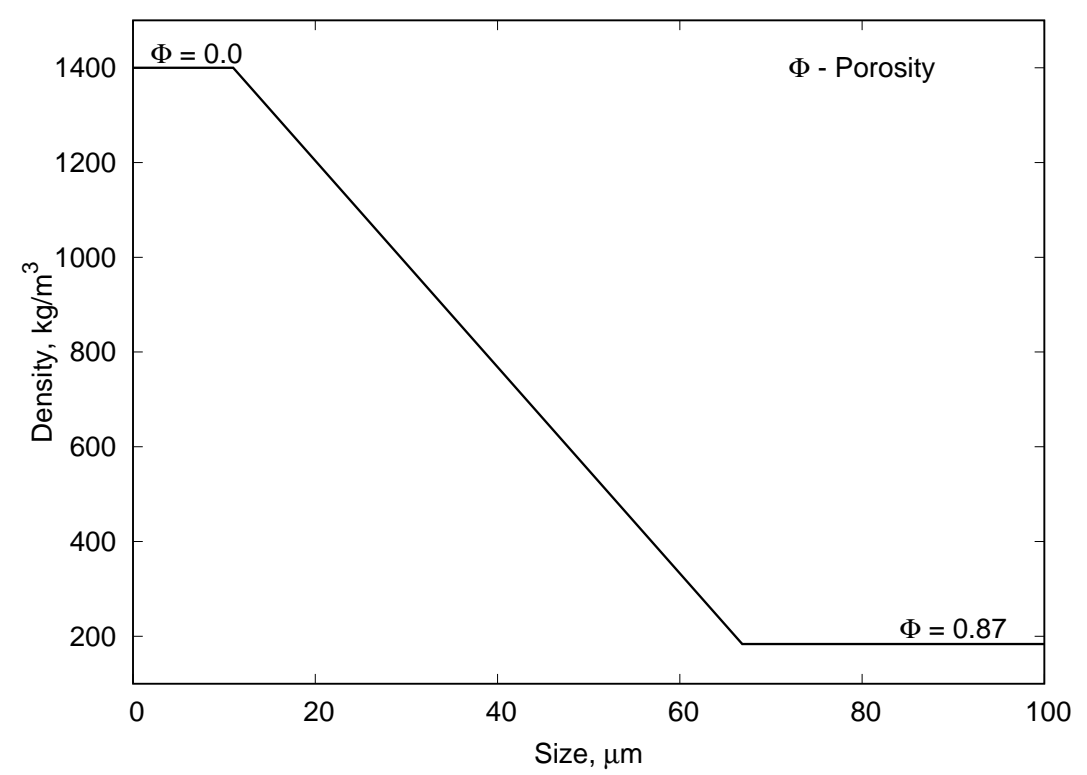

Figure 2. Variation of density of chunk of fibers with respect to size of the spherical control volume

\section{Results and Discussions}

\section{IV.A. Flow field}

The HYMETS arc-jet environment is numerically simulated using KATS - CFD by Düzel et al. ${ }^{30}$ The upstream properties are extracted from this solution and are used as boundary conditions to compute the flow field around the wedge. The perimeter of the wedge is extracted from the images taken during the experiments. The converged solution of Mach 5 flow over the wedge sample is computed using properties listed in Table 1, and the Mach contour of the solution is illustrated in Fig. 3.

\section{IV.B. Verification}

For verification, random points from a known trajectory are taken, and the reconstruction method is employed. Fig. 4 illustrates the results of the verification procedure. Here, the lines indicate the simulated trajectory, plus symbols indicate the flight time of points relative to experimental points, shown in circle 
Table 1. Uniform flow conditions for Mach 5 Air flow

\begin{tabular}{ccccccccccc}
\hline \hline$\rho_{f}\left(\mathrm{~kg} / \mathrm{m}^{3}\right)$ & $U_{\infty}(\mathrm{m} / \mathrm{s})$ & $T_{t r_{\infty}}(\mathrm{K})$ & $T_{v e_{\infty}}(\mathrm{K})$ & $T_{w}(\mathrm{~K})$ & $Y_{\mathrm{Ar}}$ & $Y_{\mathrm{N}_{2}}$ & $Y_{\mathrm{O}_{2}}$ & $Y_{\mathrm{NO}}$ & $Y_{\mathrm{N}}$ & $Y_{\mathrm{O}}$ \\
\hline $1.02 \times 10^{-3}$ & 3338.22 & 957.07 & 4892.83 & 2360 & 0.068 & 0.699 & $4.6 \times 10^{-4}$ & $1.3 \times 10^{-5}$ & 0.0154 & 0.217 \\
\hline \hline
\end{tabular}

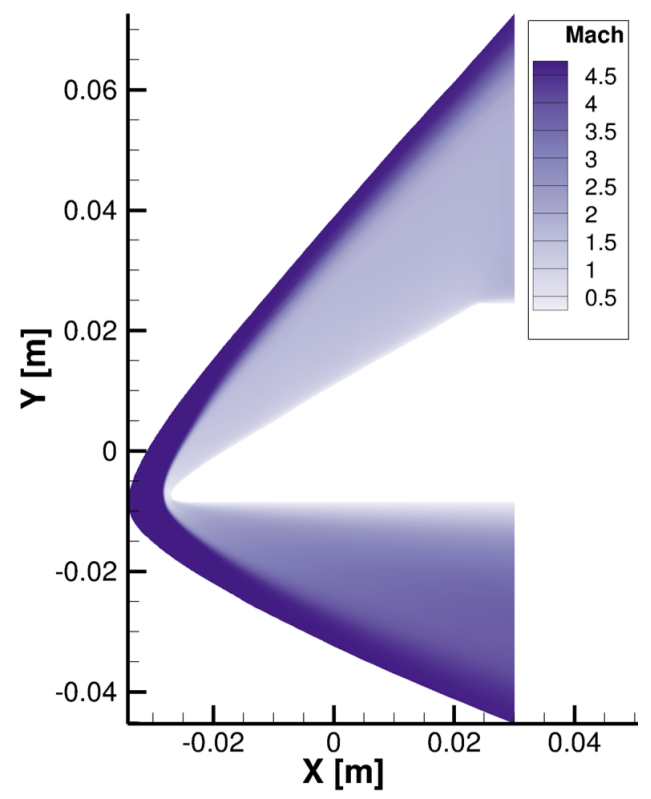

Figure 3. Mach contour for hypersonic flow around wedge based on NASA HYMETS test conditions 
symbols. Table 2 shows the change in the cost function with iterations. It can be seen that as iterations increase, the simulated trajectory moves closer to experimental points, as illustrated in Fig. 4 . It is to be noted that the simulated trajectory for iteration 7 and 8 is the same. However, the time of flight points matches the experimental points for iteration 8, as shown in the zoomed plot of Fig. 4.

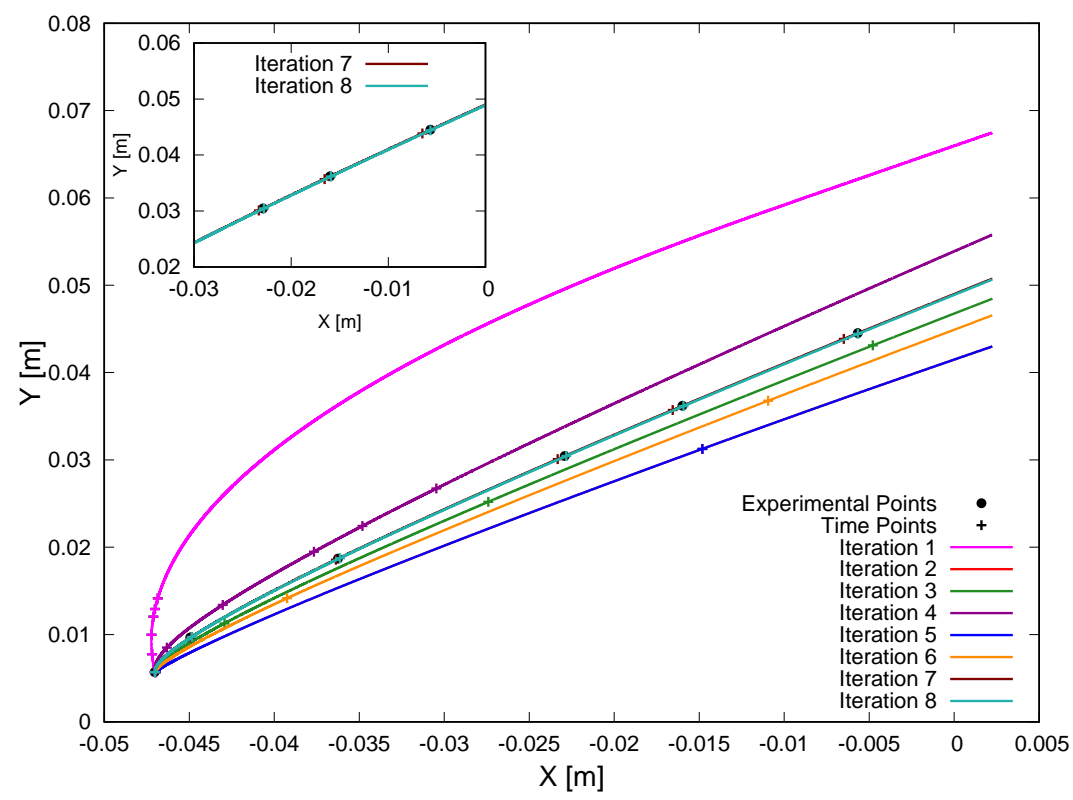

Figure 4. Variation of simulated trajectories with respect to iteration for reconstruction

Table 2. Iteration vs. Cost function

\begin{tabular}{cc}
\hline Iteration \# & Cost function \\
\hline 1 & $5.2524 \mathrm{E}-003$ \\
2 & $6.6175 \mathrm{E}-003$ \\
3 & $4.7386 \mathrm{E}-003$ \\
4 & $1.8897 \mathrm{E}-003$ \\
5 & $3.2606 \mathrm{E}-003$ \\
6 & $2.8329 \mathrm{E}-003$ \\
7 & $2.0361 \mathrm{E}-006$ \\
8 & $5.4174 \mathrm{E}-008$ \\
\hline
\end{tabular}

\section{IV.C. Numerical reconstruction}

The numerical reconstruction is performed by adapting the particle's initial velocity components and size to match the experimental trajectory. However, for these preliminary results, the reconstruction is only performed starting from the first point of the trajectory. The numerical trajectory is not backtracked to the surface of the wedge. The reconstructed trajectories pass through experimental points and match them both in position and the time of flight. Figure 5 illustrates the results for 11 of the 600 reconstructed trajectories. In the plot, both the reconstructed trajectory and the experimental points are shown.

The sizes of the particles extracted by reconstructing 600 experimental trajectories are illustrated in Fig. 6. The spalled particles' trajectories can be divided into three types: those ejecting from the top inclined surface, those ejecting from the bottom, near the leading edge, and those ejecting from the bottom, further along, the edge of the wedge sample. All three types of trajectories are shown in Fig. 5. The reconstruction results show that the size of the particle depends on the type of trajectory. The sizes of the 


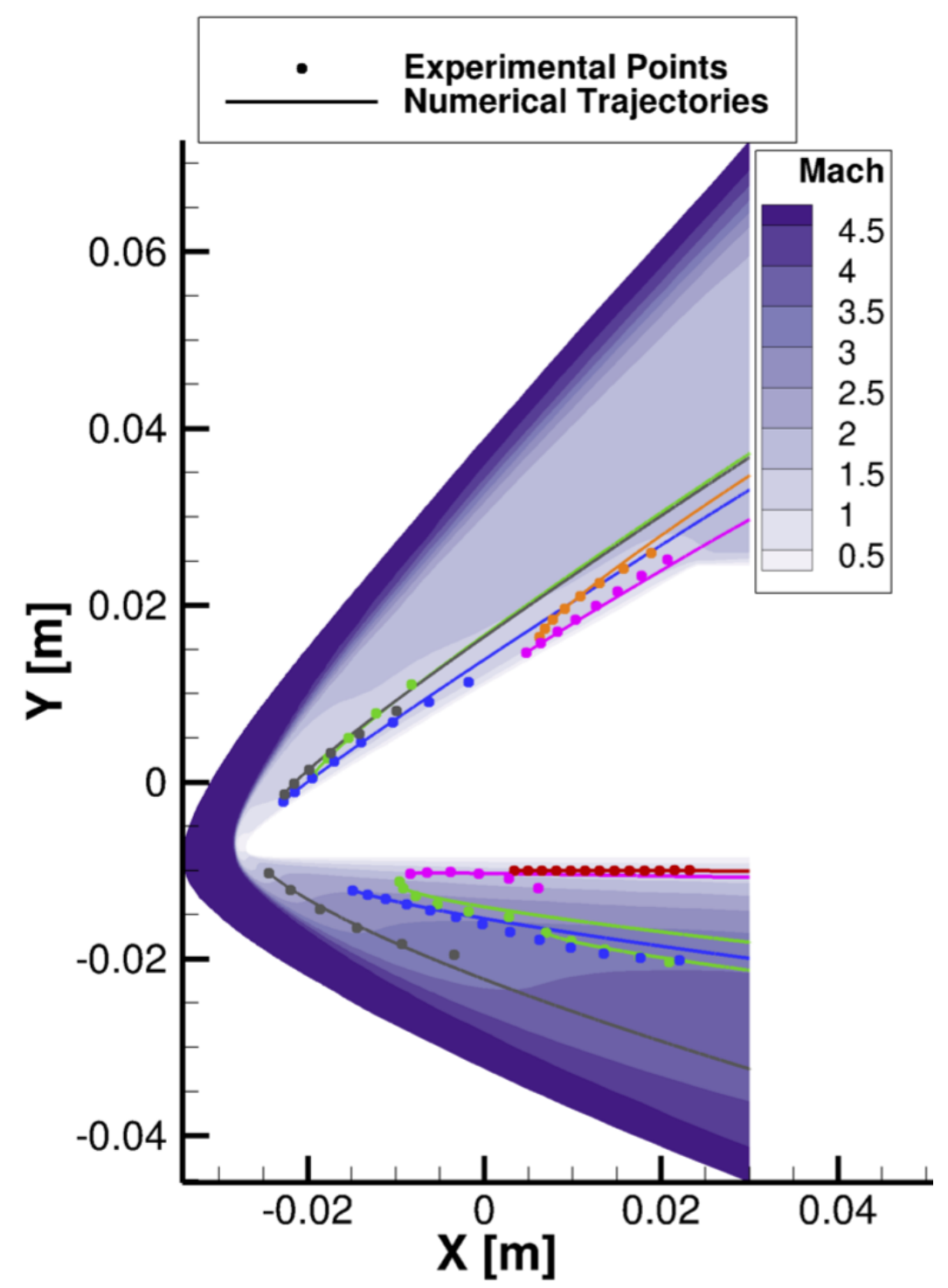

Figure 5. Numerical and experimental reconstructed trajectories 


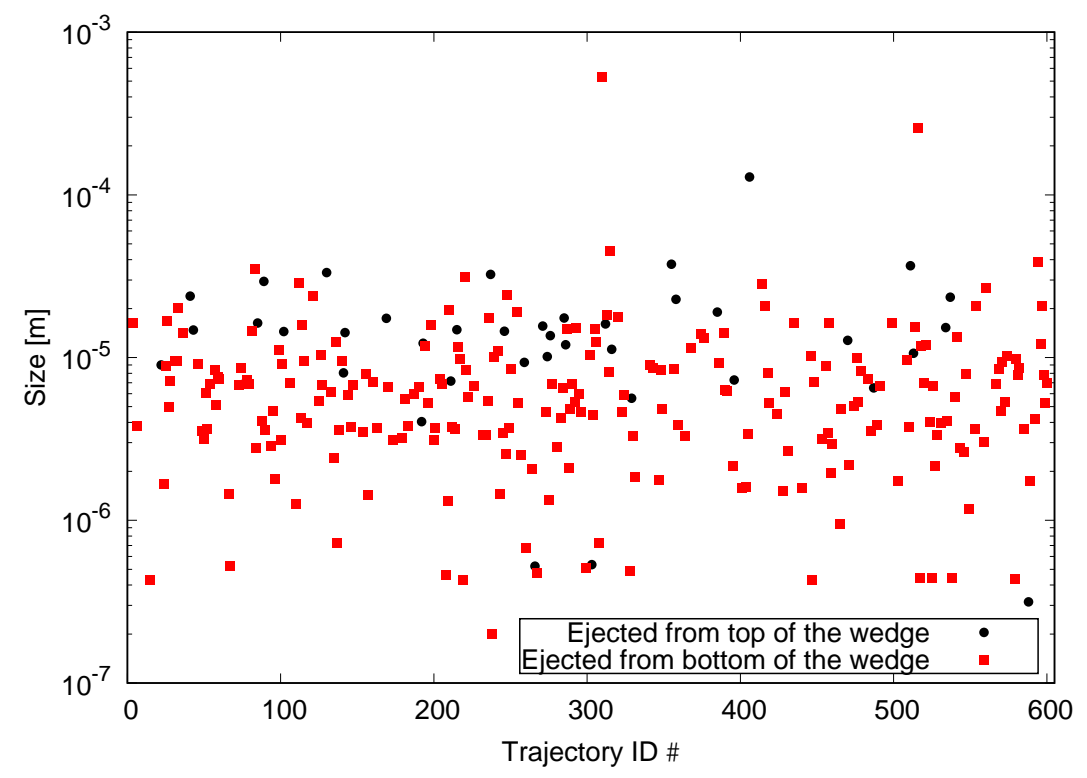

Figure 6. Particles sizes obtained from numerical reconstruction results

particles ejected from the top of the wedge, indicating the first type of trajectory, are consistent roughly at a value of $10 \mu \mathrm{m}$. On the other hand, the sizes of particles ejected from the bottom of the wedge vary between $0.1 \mu \mathrm{m}$ and $1000 \mu \mathrm{m}$. Among the ones ejected from the bottom of the wedge, the particles following the second type of trajectory are bulkier, and their sizes range between $10 \mu \mathrm{m}$ and $1000 \mu \mathrm{m}$. The particles traversing the third type of trajectory are relatively smaller compared to other types and range between $0.1 \mu \mathrm{m}$ and $1 \mu \mathrm{m}$.

The probability distribution function of particle sizes extracted from the reconstruction results are shown in Figure 7. It can be seen that the mean of the particle sizes is around $5.81 \times 10^{-6}$ with standard deviation and median at $1.81 \times 10^{-5}$ and $3.34 \times 10^{-6}$, respectively. These results are within the same range as the ones obtained by Price et al. ${ }^{11,12}$ using a different technique on similar experimental data.

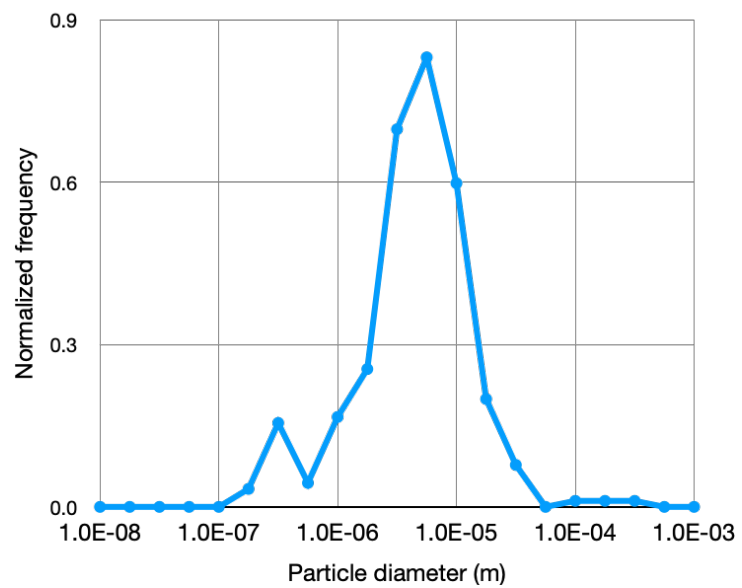

(a) Size frequency using $1 \times 10^{0.25}$ size bins

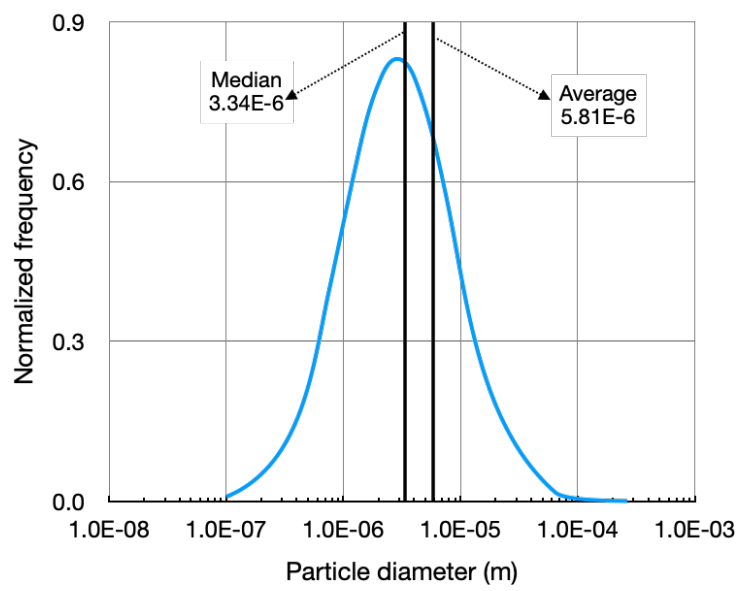

(b) Normal distribution fit of the results

Figure 7. Distribution of particle sizes extracted from numerical reconstruction 


\section{Conclusion}

It is essential to evaluate the effect of spallation phenomena compared to other ablative mechanisms to determine its significance. Therefore, numerical reconstruction of particle trajectories was employed on experimental data to estimate the mass loss caused by spallation phenomena. The perimeter of the test sample's scanned image from the experiments was extracted and used as a sample to compute a Mach 5 flow field around it using KATS-CFD.

The reconstruction was performed for a limited number of trajectories, and the simulated trajectory is reconstructed from the first point of the corresponding experimental data. It was seen that the size of the spalled particles ejected from the bottom of the wedge varies, whereas the ones ejected from the inclined surface are more uniform. On the bottom side, the reconstructed results show that the particles ejected from the leading edge are bulkier than those ejected further along the sample's side.

The reconstruction methodology will be extended by adding a back-tracking method to simulate the particle's complete path in the near future. This would also allow us to estimate the velocity at the ejection. Additional data will also be used to provide a more comprehensive and statistically reliable - understanding of the spallation phenomenon.

\section{Acknowledgements}

Financial support for this work was provided by NASA Kentucky EPSCoR Award NNX10AV39A and NASA Award NNX13AN04A. The authors would like to thank J. B. Hoagg at the University of Kentucky for his assistance with the adaptation model. The authors would also like to thank F. Panerai (University of Illinois), S. Splinter, J.G. Gragg, and W. Geouge of HYMETS at NASA Langley Research Center, and K. Price at the University of Kentucky for their support with the experiments and data. The first author would also like to thank U. Düzel, R. Fu, and S. McDaniel at the University of Kentucky for their assistance.

\section{References}

\footnotetext{
${ }^{1}$ Lundell, J. H. and Dickey, R. R., "The Response of Heat-Shield Materials to Intense Laser Radiation," AIAA 16th Aerospace Sciences Meeting, AIAA Paper 1978-138, Huntsville, Alabama, January 1978. doi:10.2514/6.1978-138

${ }^{2}$ Dickey, R. R. and Lundell, J. H., "A technique for evaluating the Jovian entry-probe heat-shield material with a gas dynamic laser," 8th International Congress on Instrumentation in Aerospace Simulation Facilities, Record (A80-29476 11-35), Institute of Electrical and Electronics Engineers, Inc., Monterey, California, September 1979, pp. 26-32.

${ }^{3}$ Lundell, J. H. and Dickey, R. R., "The CO2 gasdynamic laser as a high-intensity radiation facility," 13th Aerospace Sciences Meeting, AIAA Paper 1975-177, American Institute of Aeronautics and Astronautics, Pasadena, California, January 1975.

doi:10.2514/6.1975-177

${ }^{4}$ Park, C., Lundell, J. H., Green, M. J., Winovich, W., and Covington, M. A., "Ablation of Carbonaceous Materials in a Hydrogen-Helium Arc-Jet Flow," AIAA 18th Thermophysics Conference, AIAA Paper 1983-1561, Montreal, Canada, June 1983. doi:10.2514/3.48589

${ }^{5}$ Nicolet, W. E., Radiation Heating Environments for Jovian Entry Conditions, Vol. 49 of Progress in Astronautics and Aeronautics, American Institute of Aeronautics and Astronautics, New York, 1976, pp. 231-250.

${ }^{6}$ Kendall, R. M., Rindal, R. A., and Bartlett, E. P., "Thermochemical Ablation," AIAA Thermophysics Specialist Conference, AIAA Paper 1965-642, American Institute of Aeronautics and Astronautics, Monterey, California, September 1965. doi:10.2514/6.1965-642

${ }^{7}$ Park, C., "Stagnation-Point Ablation of Carbonaceous Flat Disks-Part I: Theory," AIAA Journal, Vol. 21, No. 11, November 1983, pp. 1588-1594. doi: $10.2514 / 3.8293$

${ }^{8}$ Park, C., "Stagnation-Point Ablation of Carbonaceous Flat Disks-Part II: Experiment," AIAA Journal, Vol. 21, No. 12, December 1983, pp. 1748-1754. doi: $10.2514 / 3.8319$

${ }^{9}$ Park, C. and Balakrishnan, A., "Ablation of Galileo Probe Heat-Shield Models in a Ballistic Range," AIAA Journal, Vol. 23, No. 2, February 1985, pp. 301-308. doi: $10.2514 / 3.8910$

${ }^{10}$ Bailey, S. C. C., Bauer, D., Panerai, F., Splinter, S. C., Danehy, P. M., Hardy, J. M., and Martin, A., "Experimental analysis of spallation particle trajectories in an arc-jet environment," Experimental Thermal and Fluid Science, Vol. 93, May 2018, pp. 319-325. doi:10.1016/j.expthermflusci.2018.01.005

${ }^{11}$ Price, K. J., Bailey, S. C. C., Panerai, F., Hardy, J. M., Borchetta, C. G., and Martin, A., "Analysis of spallation products
} 
using arc-jet experiments," 49th Thermophysics Conference, AIAA Paper 2020-3279, Reno, NV, June 2020.

doi:10.2514/6.2020-3279

${ }^{12}$ Price, K. J., Borchetta, C. G., Hardy, J. M., Panerai, F., Bailey, S. C. C., and Martin, A., "Arc-Jet Measurements of Low-Density Ablator Spallation," Experimental Thermal and Fluid Science, 2020, Submitted.

${ }^{13}$ Price, K. J., Hardy, J. M., Borchetta, C. G., Bailey, S. C. C., Panerai, F., and Martin, A., "Spallation particle size analysis resulting from arc-jet experiments," 58th AIAA Aerospace Sciences Meeting, AIAA Paper 2020-1707, Orlando, FL, Jan 2020 .

doi:10.2514/6.2020-1707

${ }^{14}$ Davuluri, R. S. C., Zhang, H., and Martin, A., "Numerical Study of Spallation Phenomenon in an Arc-Jet Environment," Journal of Thermophysics and Heat Transfer, Vol. 30, No. 1, January 2016, pp. 32-41. doi:10.2514/1.T4586

${ }^{15}$ Davuluri, R. S. C., Zhang, H., and Martin, A., "Effects of spalled particles thermal degradation on a hypersonic flow field environment," 54th AIAA Aerospace Sciences Meeting, AIAA Paper 2016-0248, San Diego, California, January 2016. doi:10.2514/6.2016-0248

16 "OpenPTV Web page," http://www.openptv.net, 2014.

${ }^{17}$ Davuluri, R. S. C., Modeling of spallation phenomenon in an arc-jet environment, Master's thesis, University of Kentucky, Lexington, Kentucky, August 2015.

${ }^{18}$ Roy, C. J., Smith, T. M., and Ober, C. C., "Verification of a Compressible CFD Code using the Method of Manufactured Solutions," 32nd AIAA Fluid Dynamics Conference and Exhibit, AIAA Paper 2002-3110, St. Louis, Missouri, June 2002. doi:10.2514/6.2002-3110

${ }^{19}$ Davuluri, R. S. C., Bailey, S. C. C., Tagavi, K. A., and Martin, A., "A drag-coefficient model for Lagrangian particle dynamics relevant to high-speed flows," International Journal of Heat and Fluid Flow, Vol. 87, February 2021. doi:10.31224/osf.io/538my

${ }^{20}$ Martin, A., Bailey, S. C. C., Panerai, F., Davuluri, R. S. C., Zhang, H., Vazsonyi, A. R., Lippay, Z. S., Mansour, N. N., Inman, J. A., Bathel, B. F., Splinter, S. C., and Danehy, P. M., "Numerical and experimental analysis of spallation phenomena," CEAS Space Journal, Vol. 8, No. 4, December 2016, pp. 229-236.

doi:10.1007/s12567-016-0118-4

${ }^{21}$ Zhang, H., High Temperature Flow Solver for Aerothermodynamics Problems, Ph.d. thesis, University of Kentucky, Lexington, Kentucky, August 2015.

${ }^{22}$ Balay, S., Gropp, W. D., McInnes, L. C., and Smith, B. F., "Efficient Management of Parallelism in Object Oriented Numerical Software Libraries," Modern Software Tools in Scientific Computing, edited by E. Arge, A. M. Bruaset, and H. P. Langtangen, Birkhäuser Press, Boston, MA, 1997, pp. 163-202. doi:10.1007/978-1-4612-1986-6_8

${ }^{23}$ Balay, S., Brown, J., Buschelman, K., Eijkhout, V., Gropp, W. D., Kaushik, D., Knepley, M. G., McInnes, L. C., Smith, B. F., and Zhang, H., "PETSc Users Manual," Tech. Rep. ANL-95/11 - Revision 3.3, Argonne National Laboratory, 2012.

${ }^{24}$ Balay, S., Abhyankar, S., Adams, M. F., Brown, J., Brune, P., Buschelman, K., Eijkhout, V., Gropp, W. D., Kaushik, D., Knepley, M. G., McInnes, L. C., Rupp, K., Smith, B. F., and Zhang, H., "PETSc Web page," http://www.mcs.anl.gov/petsc, 2014.

${ }^{25}$ Karypis, G. and Kumar, V., "A Fast and High Quality Multilevel Scheme for Partitioning Irregular Graphs," SIAM Journal on Scientific Computing, Vol. 20, No. 1, January 1998, pp. 359-392. doi:10.1137/S1064827595287997

${ }^{26}$ Walker, D. W. and Dongarra, J. J., "MPI: a standard message passing interface," Supercomputer, Vol. 12, No. 1, December 1996, pp. 56-58.

${ }^{27}$ Li, Z., Zhang, H., Bailey, S. C. C., Hoagg, J. B., and Martin, A., "A data-driven adaptive Reynolds-averaged NavierStokes k- $\omega$ model for turbulent flow," Journal of Computational Physics, Vol. 345, September 2017, pp. 111-131. doi:10.1016/j.jcp.2017.05.009

${ }^{28}$ Davuluri, R. S. C. and Martin, A., "Development of a correlation between porosity of the material and size of the spherical particle ejected from it," Unpublished.

${ }^{29}$ Panerai, F., White, J. D., Cochell, T. J., Schroeder, O. M., Mansour, N. N., Wright, M. J., and Martin, A., "Experimental measurements of the permeability of fibrous carbon at high-temperature," International Journal of Heat and Mass Transfer, Vol. 101, October 2016, pp. 267-273. doi:10.1016/j.ijheatmasstransfer.2016.05.016

${ }^{30}$ Ümran Düzel, Schroeder, O. M., Zhang, H., and Martin, A., "Computational Prediction of NASA Langley HYMETS Arc Jet Flow with KATS," 2018 AIAA Aerospace Sciences Meeting, AIAA Paper 2018-1719, American Institute of Aeronautics and Astronautics, Kissimmee, Florida, January 2018.

doi:10.2514/6.2018-1719 